\title{
Product Failure: Severity and Locus of Causality Effects on Brand Evaluations
}

\author{
Sujin Song, Dan A. Sheinin, and Sukki Yoon
}

\begin{abstract}
Although product failures cause marketers and consumers to suffer substantial damages and losses, failures are often beyond control. Building on defensive attribution literature, this study experimentally investigates how locus of causality and outcome severity of product failure interactively shape consumers' brand evaluations. Findings show that following a product failure experience, consumers respond with the lowest brand evaluation for a brand-caused failure, a higher brand evaluation for a natural disaster-caused failure, and the highest brand evaluation for a consumer-caused failure. Outcome severity moderates the effects; however, when the failure causes severe outcomes, positive brand evaluation deteriorates for the consumer-caused failure but not for the brand- and natural disastercaused failure. In addition, brand-blame attribution mediates the relationships.
\end{abstract}

\footnotetext{
S. Song $(\square)$

Korea University, Seoul, South Korea

e-mail: songsj@korea.ac.kr

D.A. Sheinin

University of Rhode Island, Kingston, RI, USA

e-mail: dsheinin@uri.edu

S. Yoon

Bryant University, Smithfield, RI, USA

e-mail: syoon@bryant.edu

L. Petruzzellis, R.S. Winer (eds.), Rediscovering the Essentiality of Marketing, Developments in Marketing Science: Proceedings of the Academy of Marketing Science, DOI 10.1007/978-3-319-29877-1_20
} 